\title{
RatingScaleReduction package: stepwise rating scale item reduction without predictability loss
}

\author{
by Waldemar W. Koczkodaj, Feng Li, and Alicja Wolny-Dominiak
}

\begin{abstract}
This study presents an innovative method for reducing the number of rating scale items without predictability loss. The "area under the receiver operator curve" method (AUC ROC) is used for the stepwise method of reducing items of a rating scale. RatingScaleReduction R package contains the presented implementation. Differential evolution (a metaheuristic for optimization) was applied to one of the analyzed datasets to illustrate that the presented stepwise method can be used with other classifiers to reduce the number of rating scale items (variables). The targeted areas of application are decision making, data mining, machine learning, and psychometrics.
\end{abstract}

Keywords: rating scale, receiver operator characteristic, ROC, AUC, scale reduction.

\section{Introduction}

Rating scales are designed to gather data and rate an entity (objects of concepts). Rating scales are also called assessment scales. In our study, we use "the scale" when no ambiguity takes place. Probably, the most significant and frequently used rating scales are exams or tests (e.g., Ontario Driver's test with 40 multiple-choice questions). Some rating scales use values " 1 to 10 " but five "stars" are gaining popularity for the online reviewing of goods or services. Yes/no answers may also be used for answers in rating scales. Number of questions (called items in the rating scale terminology) may also drastically differ from scale to scale. Numerous rating scales have over 100 items to rate but one item rating scale is also useful for rating the customer's satisfaction with goods or services. An example of a popular rating scale is the intelligence quotient (IQ). It is a total score derived from several standardized tests designed to assess human intelligence.

Sometimes, the scale is called a survey or a questionnaire. A questionnaire is a tool for data gathering and may not be used for a rating. A survey may not necessarily be conducted by questionnaires and usually does not rate anything. Its goal is to gather data. Some surveys may be conducted by interviews or extracted by Internet agents with or without our consent or knowledge. The important distinction of rating scales from questionnaires and surveys, is that the rating scales are used for assessments. It means that rating scales are expected to have an outcome making them classifiers (in the terminology of statistics and machine learning). The scale term in the rating scale has the meaning as in "the scale of disaster" hence this study assumes that:

$$
[\text { ratingscale }]=[\text { dataframe }]+[\text { assessment }]=[\text { classifier }]
$$

The "assessment" procedure must be in place for a questionnaire or survey to become a rating scale. The assessment procedure may be as complex as the imagination of their authors but most psychiatric rating scales use a simple summation. The simple summation has been used in the first examples. The simple summation can be replaced by, for example, the assignment of weights. In our example 2, we computed weights by the differential evolution (using R packagbe DEval). As expected, it has not influenced the reduction, but it has improved the predictability rate.

"A picture is worth a thousand words" hence Fig. 1 has been used to illustrate ratings scales which are used in many examinations and ratings of various products.

Figure 1: Rating scale example

The recent popularity of rating scales is due to various "Customer Reviews" on the Internet where five stars are often used instead of ordinal numbers. Rating scales are predominantly used to express our subjective assessments such as "on the scale 1 to 5, express your preference" by one selection of: "strongly agree to strongly disagree" with 3 as "neutral" preference. Using a slider implementation (as in Fig. 1) gives us the flexibility. It is known as a graphic or continuous rating scale. 


\section{Terminology issue}

In our study, we decided to use the data mining (DM) terminology having in mind that regardless of the scientific discipline, the ultimate goal is to predict the outcome of future examples to make a decision. Statistical observations are called examples in DM terminology and items in the rating scale terminology. Statistics uses variables. $\mathrm{R}$ project uses attributes and the data frame with all attributes in columns and examples in rows creates an attribute matrix. The additional validation variable is called the decision vector or simply, the decision. In psychiatry, the decision may be "is the patient sick with depression or not." In case of Ontario Driver's test, it is: "do we give a passing grade or not?" The list of application is endless as decision making is of considerable importance for everyone. We also have introduced a new term: "gray examples" for two examples having identical values on all attributes but belonging to two different classes. Evidently, such situation should not occur in the ideal situation but uncertainty in data or imperfect data need to be handled in practice. The gray color is intermediate between black and white and our two examples belong to both classes. One example may be replicated $m$ times. Replication of examples would deviate computations and should be detected and removed.

\section{Rating scale reduction}

Large rating scales discourage respondents from completing them. It is not unusual to have most answers as random numbers at the end of a long rating scale. It seems that the first successful rating scale reduction took place in Velden M. \& Clark (1979) by the psychophysical model by means of signal detection theory (SDT). However, the last paragraph which served as the optional Conclusions at that time:

It may be concluded that due to the psychophysical difference between the SOT discrimination and the common rating situation, signal detection type reduction of such rating data does not allow interpretation of resulting values as unbiased psychological distances. To avoid misinterpretations, it might be worthwhile not to use the SDT notation for indices derived from psychological rating data.

may be perceived as a risky proposition. For many rating scales (e.g., written academic exams), arbitrary (rarely, computed) weights are applied for some or all items (questions) to improve the overall rating. The proposed method respects it and takes into account only the final "total" (obtained by whatever method or procedure it is established). Formally speaking, the "total" is a metric. The rating scale with metric can be regarded as a classifier for classifying subjects by the rating scale (e.g., sick or not in case of medicine). In Koczkodaj et al. (2017), the stepwise algorithm is proposed for the reduction of the rating scale items by using a metric computed from the confusion matrix by AUC of ROC. The proposed algorithm is restricted to the dichotomous (binary) decision making by the supervised learning approach. Our heuristic algorithm is addressed in Section 2.2. In practice, it is the most needed type of decision (e.g., go/stop, left/right, alive/dead, passed/failed, etc.). There are many ways of transforming general data into dichotomous data to be useful.

A dichotomous (binary) rating scale groups observations (examples) into two categories based on the knowledge about the classified subject. The knowledge is the external assessment since it is the case of supervised learning. If, for example, the division into two groups is "sick/not sick", it is necessary to know if the patient was indeed sick or not by the opinion of an MD to be able to screen (classify) future patients, unnecessarily taking time of usually busy MDs.

\section{Heuristic algorithm and rating scale stepwise reduction procedure}

ROC method and corresponding AUC is a well known technique to asses the classifier performance. Both ROC and AUC concepts are well addressed by Fawcett (2004). They are implemented by many R packages including: pROC (Robin et al., 2011) and ROCR (Sing et al., 2005). There is also one interesting web application easyROC (Goksuluk et al., 2016) giving possibility to compute the confusion matrix and plot the curve on-line. The RatingScaleReduction package expands this analysis to carry out the procedure of rating scale reduction. The main function of the package is based on the procedure described in Koczkodaj et al. (2017).

In computer science and mathematical optimization, a heuristic is a technique (or method) designed for solving a problem by finding an approximate solution when classic methods fail to find the exact solution. Often, finding such a method is achieved by trading completeness, accuracy, or optimality, for the speed. However, most heuristics are designed to find an approximate solution of NP-complete problems (NP stands for "nondeterministic polynomial time"). In layman's terms, an 
infinite computing time is needed to find an exact solution for an NP-complete problem hence a simpler (and usually approximated) solution needs to be accepted.

Evidently, heuristic algorithms produce "good enough"solutions. They are usually not the optimal solution but "good enough" is nearly always better than none. For example, the traveling salesman problem (TSP), often formulated as find the shortest possible route to visit each city once only and return to the origin city. It cannot be computed even for 50 cities by verifying all possible combinations since the total number of such permutations is estimated to $O\left(n^{2} 2^{n}\right)$ although a little bit more optimistic estimations are suspected to exist. By using heuristics, we can solve TSP for millions of cities with the accuracy of a small fraction of $1 \%$.

Heuristic algorithm is a frequently used misnomer. If it is "heuristic", it is not an algorithm and if it is algorithm, it is not a heuristic. However, many heuristics are expressed (written) the same way as algorithms but such "algorithms" do not have well established scientific foundations. Instead, they are based on observations, experience, or even intuition. Some heuristic algorithms may become algorithms. As time passes, we gather more and more evidence and such evidence may lead to finding a theory.

In rating scale reduction problem, the number of possible combinations for a rating scale with 100 items is a "cosmic number" hence the complete search must be ruled out. Certainly, the results need to be verified and used only if the item reduction is substantial. Computing the AUC of ROC for all items is the basis for our heuristic. Common sense dictates that the contribution of the individual items to the overall value of AUC of ROC needs to be somehow utilized. In the RatingScaleReduction, the implemented algorithm (when reduced to its minimum) uses a loop for all attributes (with the class excluded) to compute AUC. Subsequently, attributes are sorted in the ascending order by AUC. The attribute with the largest AUC is added to a subset of all attributes (evidently, it cannot be empty since it is supposed to be the minimum subset $S$ of all attributes with the maximum AUC). We continue adding the next in line (according to AUC) attribute to the subset $S$ checking AUC. If it decreases, we stop the procedure. There is a lot of checking (e.g., if the dataset is not empty or full of replications) involved. In a more formal way, the RSR procedure implemented in RatingScaleReduction has the general steps:

1. input: attM $[i, j]$ - attribute matrix, $i=1, \ldots, n, j=1, \ldots, m$, where $n$ - the number of examples, $m$ - the number of columns,

$D[i]$ - decision vector $i=1, \ldots, n$

2. iterate in the loop:

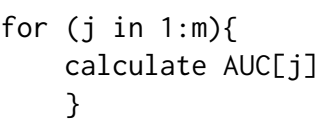

3. sort the vector $A U C[1], \ldots, A U C[m]$ in the descending order receiving a new vector

$$
\operatorname{AUCs}[1], \ldots, A U C s[m]
$$

4. create a new attribute matrix attMs $[i, j]$ with columns sorted in the descending order according to the vector:

$$
\operatorname{AUCs}[1], \ldots, A U C s[m]
$$

5. iterate in the loop:

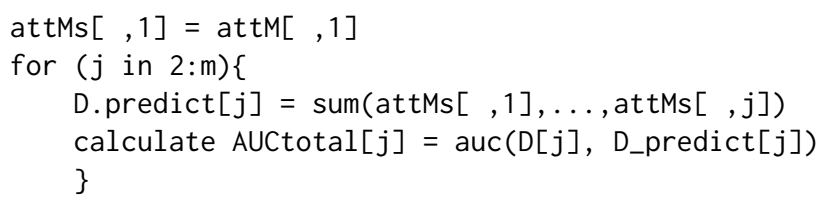

6. iterate in the loop:

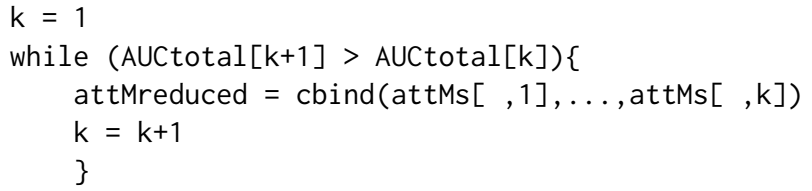

7. output: the reduced attribute matrix attMreduced generating the reduced rating scale.

First part of RSR procedure is implemented by R functions startAuc, totalAuc while the second part is the main function of the package called rsr. 


\section{RatingScaleReduction: overview of the package functions}

and shown by Fig.

The RatingScaleReduction package implements the above-stated stepwise procedure using two functions of the pROC package: roc and roc.test. The data can be the matrix or data.frame. Columns represent attributes and one column is the class with two categories: 0 or 1 (or any other two different integer or real values). The rows in data. frame represents examples. For our package, all attributes and the class must be numeric (preferably 0 or 1 ) hence some preprocessing may be needed.

There are two groups of functions available in the package. The first group is for implementing the core of the RSR algorithm:

1. startAuc (attribute, D) - compute the AUC values of every single attribute in the rating scale.

2. totalAuc (attribute, D, plotT=FALSE) - sort AUC values in the ascending order and compute AUCs of running total of first $k$ attributes, $k=1, \ldots, n$, where $n$ is the number of attributes. Setting the argument plotT as TRUE the plot of new AUC values is created. The horizontal line marks the max new AUC.

3. $\operatorname{rsr}$ (attribute, D, plotRSR=FALSE) - the main function of the package reducing the rating scale. Setting the argument plotRSR as TRUE the plot of ROC curve of the sum of attributes in reduced rating scale is created.

4. CheckAttr4Inclusion (attribute, D) - subsequently, we check the next attribute for the possible inclusion in the reduced set of attribute. It is done by maximizing AUC of all already included attributes and the attribute we have just checked. In some cases, all attributes will be included in the new set of attributes. The reduced set of one attribute may be created if there is an identifying attribute. The function CheckAttr4Inclusion tests the inclusion. It carried out a statistical test for a difference in AUC of two correlated ROC curves: ROC 1 of the sum of attributes from reduced rating scale and ROC2 of this sum plus the next ordered attribute. The function roc. test from the pROC is used and all implemented tests are available, in particular delong and bootstrap.

The package RatingScaleReduction also contains the second group of functions to support the reduction procedure. Before running the PROC1, the dataset should be analyzed to detect replicated examples (gray examples). This analysis of a dataset can be done by using functions: diffExamples and grayExamples.

1. diffExamples (attribute) - search replicated examples in the data and return the number of different examples and the number of duplicates.

2. grayExamples (attribute, D) - produce the list of pairs of examples having identical values on all attributes. The decision value and attributes are produced for every pair in the dataset, so the list clearly shows all gray examples.

In the examples presenting the capabilities of the RatingScaleReduction package, we have used the following two datasets:

1. Wine quality demonstrates how most of our package functions are used,

2. Somerville Happiness Survey for the use of differential evolution (DE) classifier.

Subsequently, we utilized our examples to demonstrate the capabilities of the RatingScaleReduction package. The full R code is available for download from https://github.com/woali/RatingScaleReduction/ blob/master/example_Rj.r.

\section{The first demonstration example: wine quality}

Wine quality dataset taken from http://archive.ics.uci.edu/ml/index. php and available in the object wineData from the RatingScaleReduction package is used in this example. It has 6497 examples and 11 attributes. The reduction is achieved by three core functions of the package. The data. frame we work on contains 11 columns with attributes and one additional column as a decision (reality).

We begin the analysis by computing AUC for all 11 individual attributes by the use of function totalAuc. Setting the argument plotT as TRUE produced a plot.

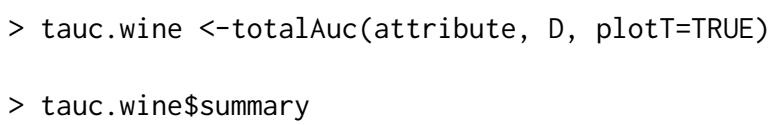




$\begin{array}{lll}\text { alcohol } & 0.6098691 & 0.6098691 \\ \text { volatile.acidity } & 0.6047200 & 0.6397657 \\ \text { fixed.acidity } & 0.5656387 & 0.6415913 \\ \text { citric.acid } & 0.5550811 & 0.6294707 \\ \text { total.sulfur.dioxide } & 0.5536676 & 0.5971653 \\ \text { sulphates } & 0.5394024 & 0.5824898 \\ \text { density } & 0.5218842 & 0.5109257 \\ \text { chlorides } & 0.5215313 & 0.5227466 \\ \text { pH } & 0.5134569 & 0.5229943 \\ \text { free.sulfur.dioxide } & 0.5052413 & 0.5237751 \\ \text { residual.sugar } & 0.4957781 & 0.5286474\end{array}$

The $\mathbf{R}$ output shows the tauc. wine $\$$ summary AUC of every single attribute in the second column, sorted in the ascending order. The running total of AUCs is in the third column. The initially selected variable (alcohol) for the first row is the attribute with the largest AUC. Subsequently, we add to it the variable with the largest AUC of the remaining attributes. The process continues while the last attribute of the scale is added.

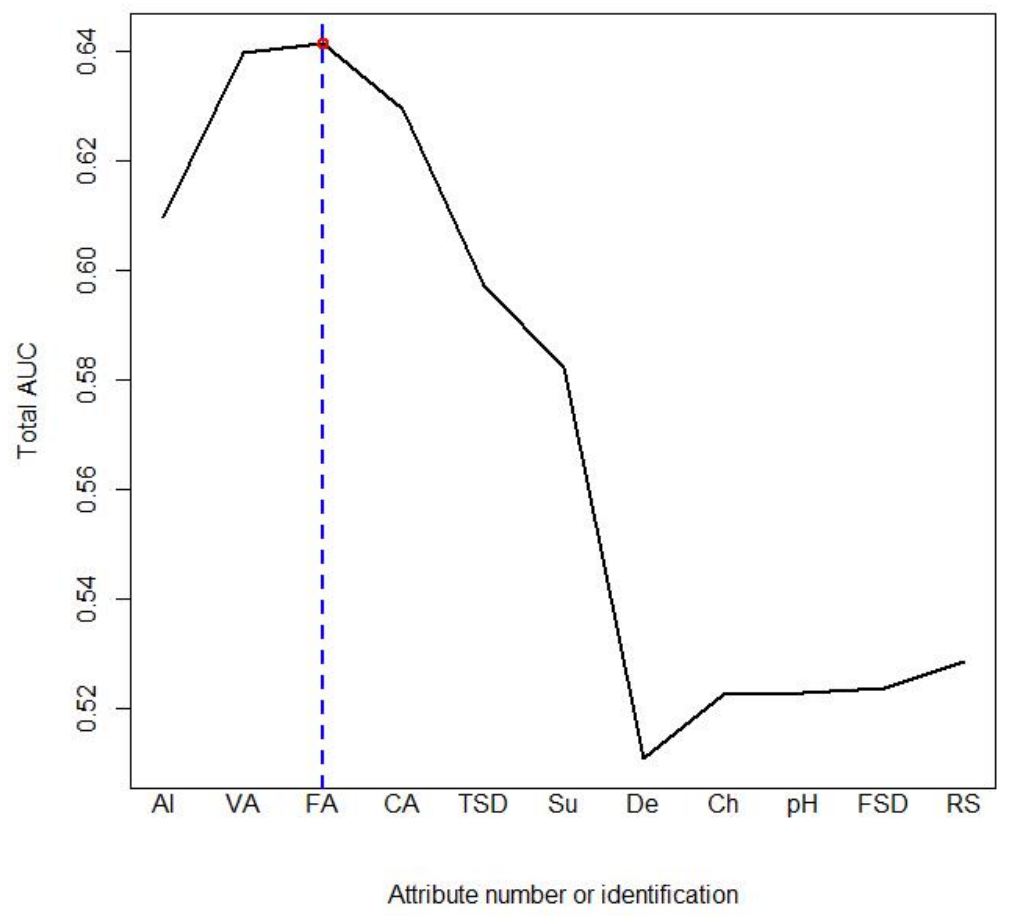

Figure 2: A stepwise AUC reduction method (example) 
Printing the value tauc. wine $\$$ i tem we receive the attribute labels in an ascending order.

$>$ tauc.wine\$item

[1] "alcohol"

[4] "citric.acid"

[7] "density"

[10] "free.sulfur.dioxide" "residual.sugar"

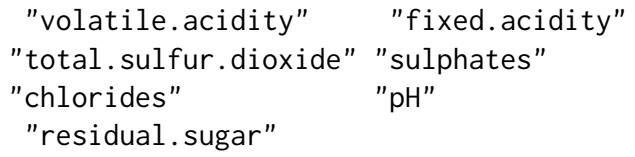

"volatile.acidity" "fixed.acidity"

As illustrated by Fig. 2, the value of AUC of the selected subset of attributes is increasing by adding the first three attributes labeled alcohol, volatile.acidity and fixed.acidity. For this reason, the reduction procedure is terminated after the first six attributes are added. The function rsr reduces the scale automatically assuming the truncation point as the attribute that first reaches the maximum AUC. AUC is a real value between 0 and 1 . It is 0.5 for random data but hardly ever reaches 1 since, in reality, there are always "gray examples" in sizable data.

$>$ rsr.wine <-rsr (attribute, D, plotRSR=TRUE)

The criteria: Stop first MAX AUC

$>$ rsr.wine\$rsr.auc

[1] $0.6098691 \quad 0.6397657 \quad 0.6415913$

$>$ rsr.wine $\$ r s r$. label

[1] "alcohol" "volatile.acidity" "fixed.acidity"

$>$ rsr.wine\$summary

AUC one variable AUC running total

alcohol $\quad 0.6098691 \quad 0.6098691$

volatile.acidity $\quad 0.6047200 \quad 0.6397657$

fixed.acidity $\quad 0.5656387 \quad 0.6415913$

Setting the rsr parameter plotRSR as TRUE the function generates the plot illustrated by Fig. 3.

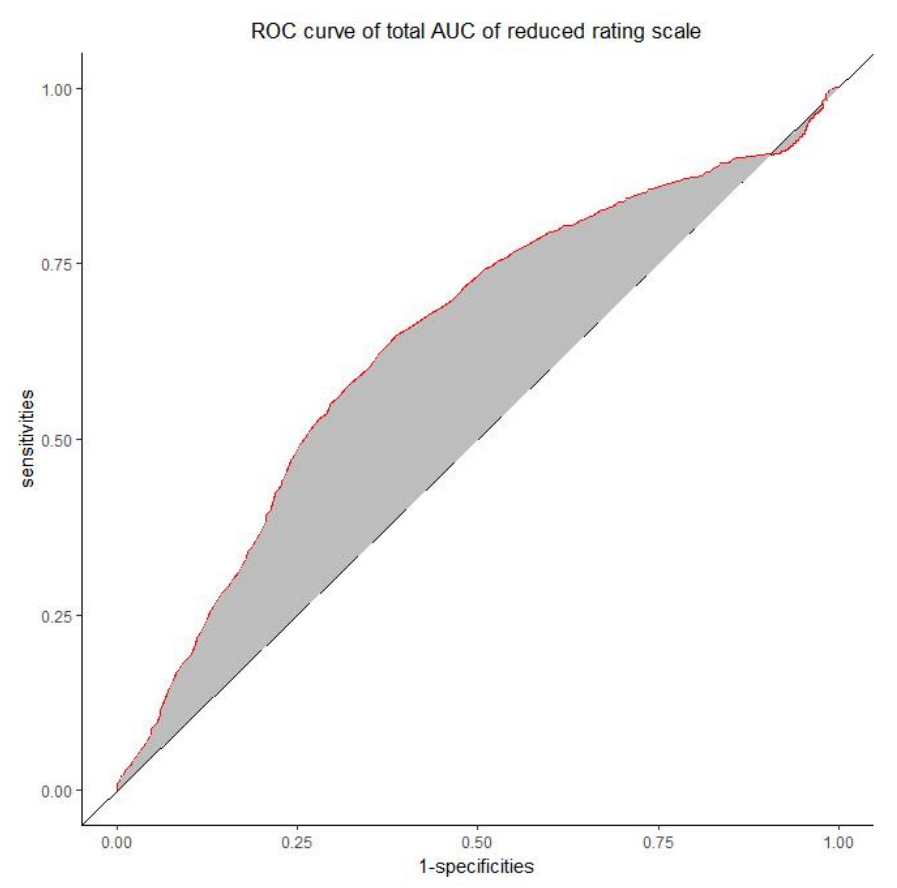

Figure 3: AUC of alcohol + volatile.acidity + fixed.acidity for Wine dataset

We assume that by selecting the "best" attribute in a loop, we are able to reduce the number of attributes for the best preventiveness. In our case, having the largest AUC is the "best" criterion. Adding the next "best" attribute to the selected attribute from the subset of the remaining attributes 
until AUC of all selected attributes decreases is the main idea of our heuristic. So far, each and every rating scale has been reduced.

\section{The second demonstration example: DEvol classifiers}

Somerville Happiness Survey (SHS) dataset has been used as the second example to demonstrate the use of Differential Evolution (DE) as a classifier to enhance the data be preprocessing. The use "survey" is warranted since it gathers "other data" but it has subscale (a part of it) for the happiness rating. This survey has been given sent out to a random sample of Somerville residents asking them to rate their personal happiness and their satisfaction with city services every second year since 2011. Every year, the survey is refined. We used data of year 2015 since this survey is the most mature. SHS dataset uses what is called a subscale. The processed survey data are available in the object SHSData in the RatingScaleReduction. It is a subscale marked s block "6" and shown by Fig. 4. The decision (class) attribute is the SHS survey question "3":

How satisfied are you with Somerville as a place to live? mapped into $0-1$ ( 0 for values less than 8 otherwise 1$)$.

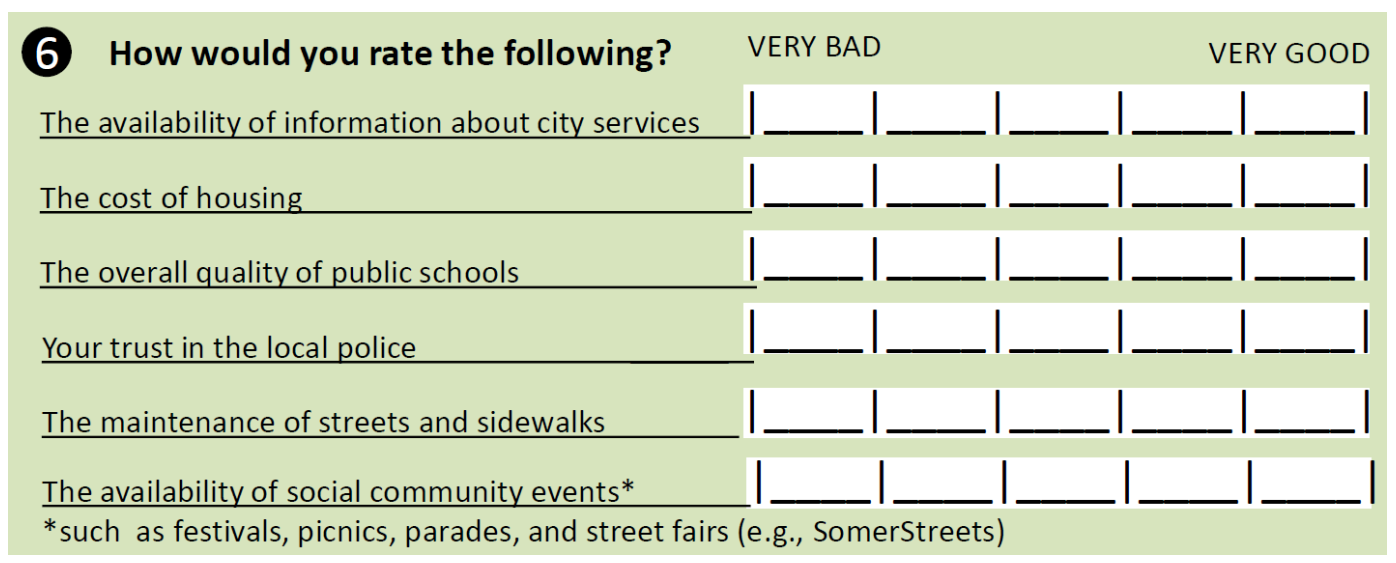

Figure 4: Survey for collecting SHS scale

For this subscale, we used Differential Evolution (DE) classifier by using DEoptim from DEoptim R package. DEoptim computes optimal weights (as a vector $w$ ) for a given data. Given data are modified by the scalar vector multiplication.

It is worth noticing that all rating scales can be improved by DEoptim. In the worse case scenario, the initial vector $[1,1, \ldots, 1]$ will remain unchanged. In our case, DEval improved the predictability measured by AUC from 0.678 to 0.789 . 
$>$ D.predict <- rowSums (attribute)

$>\operatorname{roc}(D, D . p r e d i c t$, plotROC $=$ FALSE) $\$$ auc

Area under the curve: 0.6794

The RSR procedure gives the following reduction:

$>$ rsrSum <- rsr(attribute, $D$, plotRSR $=$ TRUE)

$>$ rsrSum

$\$ r s r$.auc

[1] $0.6664868 \quad 0.6934700 \quad 0.7330005$

$\$ r s r . l a b e l$

[1] "X6" "X1" "X4"

\$summary

AUC one variable AUC running total

$\begin{array}{lll}\text { X6 } & 0.6664868 & 0.6664868 \\ \text { X1 } & 0.6542094 & 0.6934700 \\ \text { X4 } & 0.6083378 & 0.7330005\end{array}$

The results are illustrated by plots (see Fig. 5 and 6).

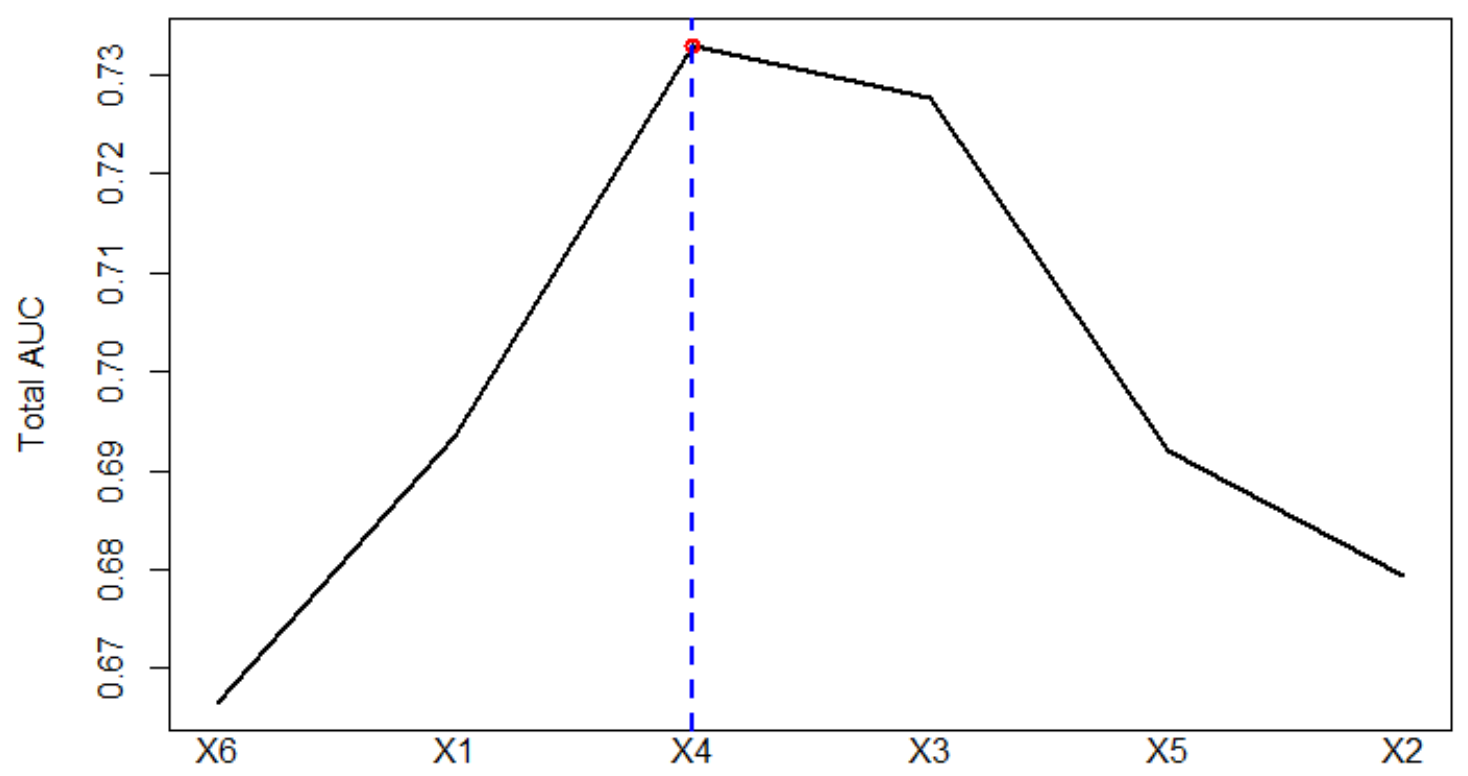

Attribute number or identification

Figure 5: Somerville happiness survey dataset

Differential evolution (DE) as a classifier In RSR procedure, we change the classifier from the simple to the weighted total. The optimal weights are received using DE. 


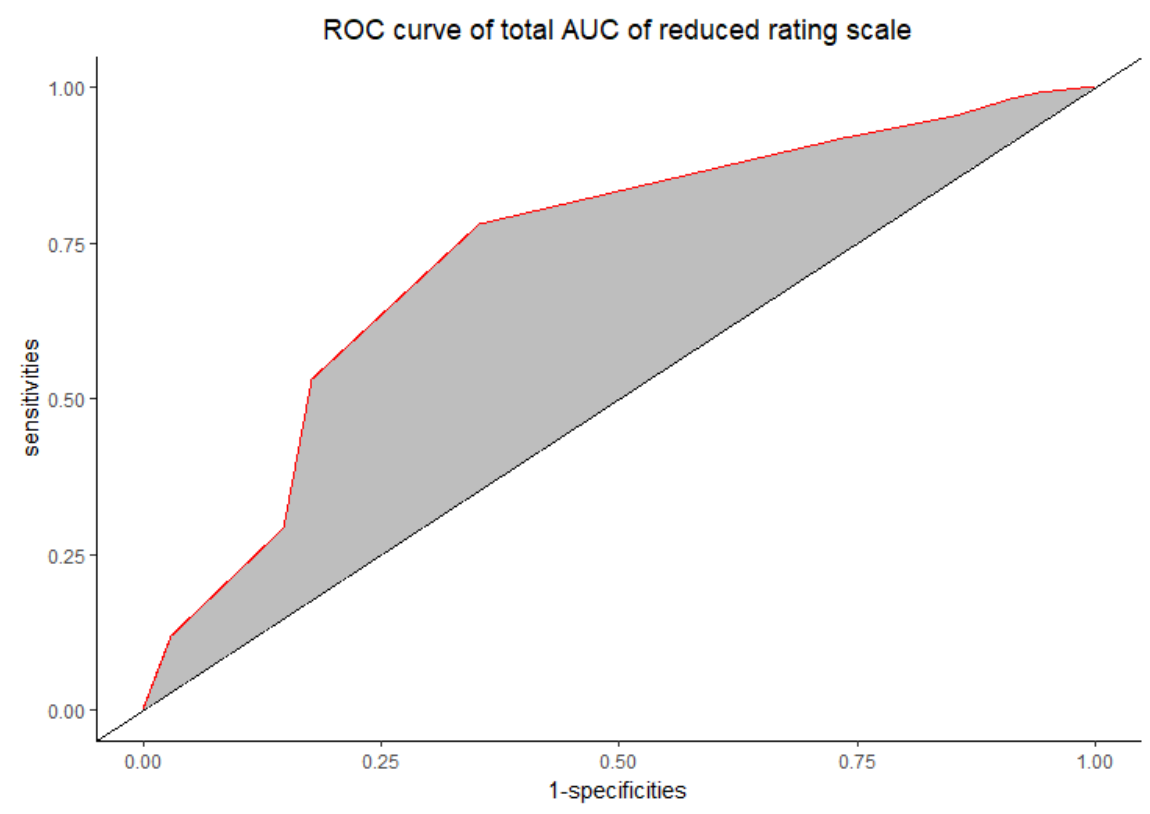

Figure 6: Somerville happiness survey dataset

The goal function is:

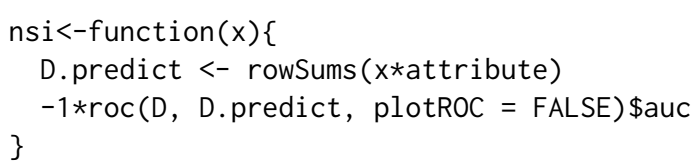

The initial lower and upper bounds, required by DE, are set to:

lower_nsi <-c $c(0.1,0.1,0.1,0.1,0.1,0.1)$

upper_nsi $<-c(3,3,3,3,3,3)$

DE optimization:

output <- DEoptim(nsi, lower_nsi, upper_nsi, DEoptim. control (itermax=10))

\#ouput the optimize result of weights

$>$ (weight.item.all <- output.all\$optim\$bestmem)

par1 par2 par3 par4 par5 par6

$\begin{array}{lllllll}1.405835 & 1.882602 & 1.758763 & 2.356450 & 1.123748 & 2.085982\end{array}$

\#all items

$>$ (aucResult <- -1*output\$optim\$bestval)

[1] 0.766055

In order to reduce the scale using DE, we have modified RSR procedure by changing D.predict formula to:

D. $\operatorname{predict}[j]=\operatorname{sum}(x[1] * \operatorname{attMs}[, 1], \ldots, x[1] * \operatorname{attMs}[, j])$ 
The sorted attribute matrix attMs $[i, j]$ we use in the loop:

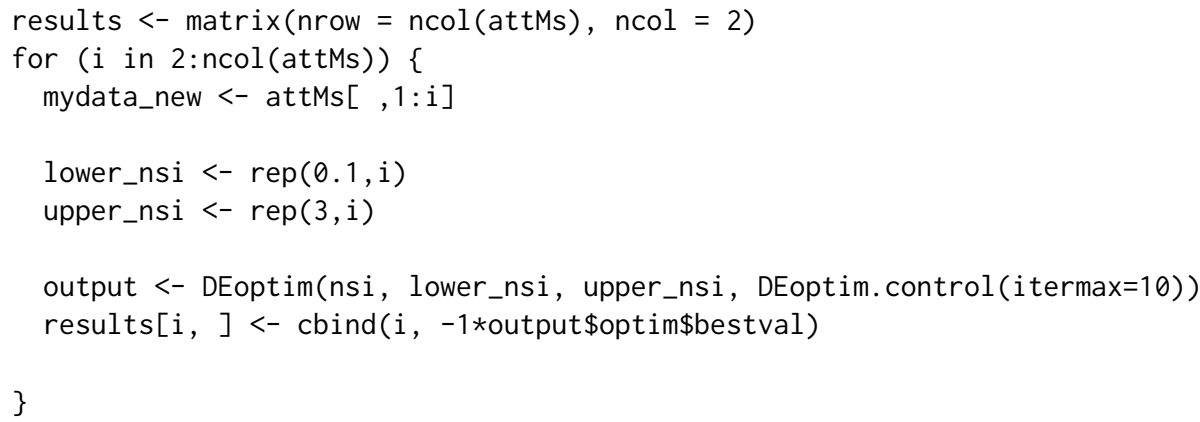

We have obtained the reduction:

$\begin{array}{lrr}\begin{array}{r}\text { results } \\ \text { number of items }\end{array} & \text { AUCtotalDE } \\ {[1,]} & 1 & 0.5226659 \\ {[2,]} & 2 & 0.6991365 \\ {[3,]} & 3 & 0.7621425 \\ {[4,]} & 4 & 0.7664598 \\ {[5,]} & 5 & 0.7251754 \\ {[6,]} & 6 & 0.7232866\end{array}$

$>$ names (attMs)

[1] "X6" "X1" "X4" "X3" "X5" "X2"

Plots are illustrated by Fig. 7 and 8

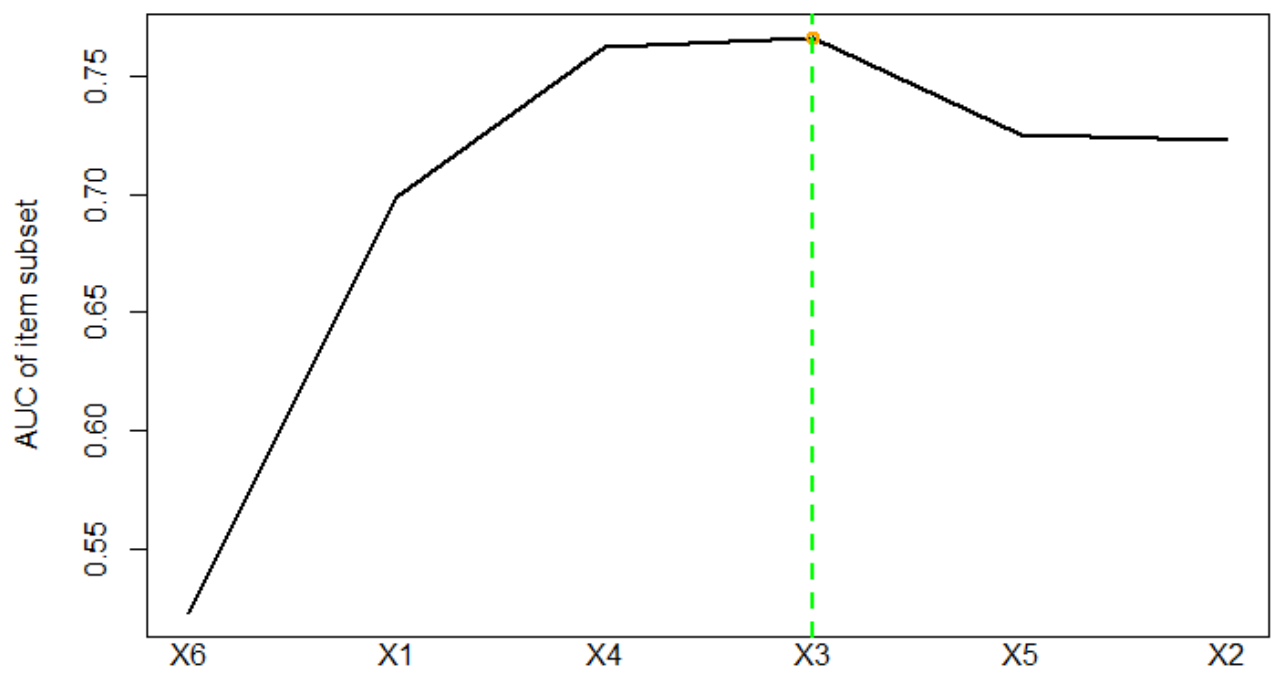

Attribute number or identification

Figure 7: Enhanced (by DE) SHS dataset

\section{The potential application targets}

Rating scales are by far more important contributors to practically all branches of applied science and engineering than we can address by this study. Most examinations for granting scientific degrees are rating scales in various shapes and forms. Simplifying them (or reducing in size) is needed if the predictability is preserved or increased. 


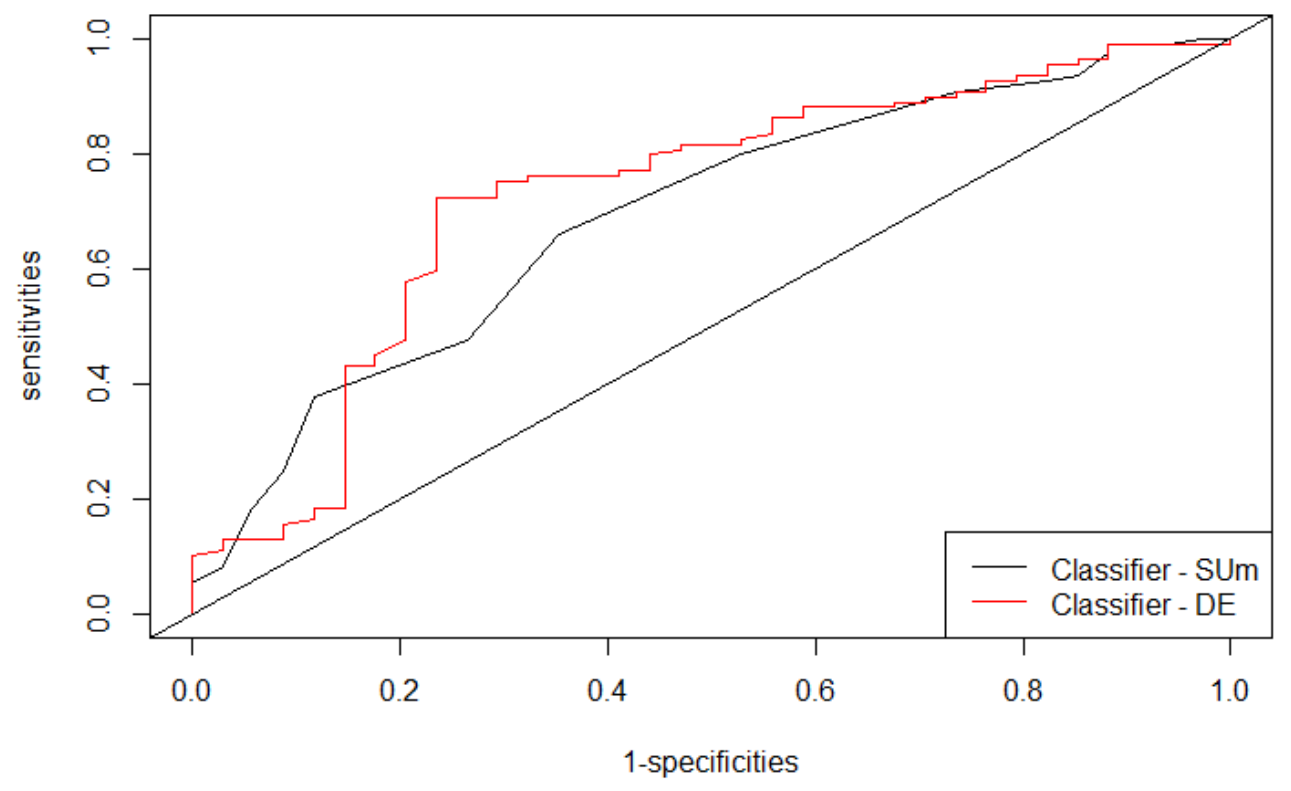

Figure 8: Enhanced (by DE) SHS dataset dataset

In bioinformatics, reporting trade-off in sensitivity and specificity, by using a Receiver Operating Characteristic (ROC) curve, is becoming a common practice. ROC plot has the sensitivity on the $y$ axis, against the false discovery rate (1- specificity) on the $x$ axis. ROC curve plot provides a visual tool to determine the boundary limit (or the separation threshold) of a subset (or a combination) of scale items for the potentially optimal combination of sensitivity and specificity. The area under the curve (AUC) of the ROC curve indicates the overall accuracy and the separation performance of the rating scale. It can be readily used to compare different item subsets. As a rule of thumb, the fewer scale items used to maximize the AUC of the ROC curve, the better.

World Health Organization estimates are included behind selected rating scales for mental disorder. Rating scales are of considerable importance for psychiatry where they are predominately used for screening patients for mental disorders such as:

- depression (see Koczkodaj et al. (2017)) which affects 60 million people worldwide according to WHO (2016),

- bipolar affective disorder (60 million people),

- dementia and cognitive impairment (47.5 million people)

- schizophrenia (21 million people),

- autism and autism spectrum disorders (e.g., Kakiashvili et al. (2012))

- addiction,

- personality and personality disorders,

- anxiety,

- ADHD;

and many other disorders.

Usually, there are many scales for each mental disorder. The most important for screening are global scales. Reducing these global rating scales makes them more usable as indicated in Koczkodaj et al. (2017). World Health Organization Media Centre reports that depression and anxiety disorders cost the global economy US $\$ 1$ trillion each year" and it is no longer a local problem.

\section{Conclusions}

The presented method has reduced the number of the rating scale items (variables) to $28.57 \%$ from the original number of items (from 21 to 6 ). It means that over $70 \%$ of collected data was unnecessary. It is 
not only an essential budgetary saving, as the data collection is usually expensive and may easily go into hundreds of thousands of dollars, but excessive data collection may contribute to data collection error increase. The more data are collected, the more errors may occur since a lack of concentration and boredom are realistic factors.

By using the proposed AUC ROC reduction method, the predictability has increased by approximately $0.5 \%$. It may seem insignificant. However, for a large population, it is of considerable importance. In fact, WHO (2016) states that: "Taken together, mental, neurological and substance use disorders exact a high toll, accounting for $13 \%$ of the total global burden."

As pointed out, rating scales are used for mental disorders. According to WHO (2016), mental disorders are becoming a global problem.

The proposed use of AUC for reducing the number of rating scale items, as a criterion, is innovative and applicable to practically all rating scales. In the worse case scenario, no reduction takes place (the number of reduced attributes is 0 ). System $\mathbf{R}$ code is posted on the Internet (RatingScaleReduction) for the general use as a $\mathbf{R}$ package. Certainly, more validation cases would be helpful and the assistance will be provided to anyone who wishes to try this method using his/her data.

Future plans include using the presented method for measuring the harmful use of the Internet and for the improvement of numerous psychiatric scales. The reduced scales can be further enhanced by the method described in Kakiashvili et al. (2012) and Koczkodaj (1996).

\section{Acknowledgments}

The first author has been supported in part by the Euro Research grant "Human Capital".

The authors would also like to express appreciation to Tiffany Armstrong (Laurentian University, Computer Science), and Grant O. Duncan, Team Lead, Business Intelligence, Integration and Development, Health Sciences North, Sudbury, Ontario, Canada) for the editorial improvements of our text and their creative comments.

\section{Bibliography}

T. Fawcett. ROC graphs: Notes and practical considerations for researchers. Machine learning, 31(1): 1-38, 2004. [p44]

D. Goksuluk, S. Korkmaz, G. Zararsiz, and A. E. Karaagaoglu. easyROC: An interactive web-tool for ROC curve analysis using R language environment. The R Journal, 2016. [p44]

T. Kakiashvili, W. W. Koczkodaj, and M. Woodbury-Smith. Improving the medical scale predictability by the pairwise comparisons method: Evidence from a clinical data study. Computer Methods and Programs in Biomedicine, 105(3):210-216, 2012. URL https: // doi .org/10.1016/ j . cmpb . 2011.09.011. $[\mathrm{p} 53,54]$

W. W. Koczkodaj. Statistically accurate evidence of improved error rate by pairwise comparisons. Perceptual and Motor Skills, 82(1):43-48, 1996. [p54]

W. W. Koczkodaj, T. Kakiashvili, A. Szymańska, J. Montero-Marin, R. Araya, and J. Garcia-Campayo, K. Rutkowski, and D. Strzałka. How to reduce the number of rating scale items without predictability loss? Scientometrics, 111:581-593, 2017. [p44, 53]

X. Robin, N. Turck, A. Hainard, N. Tiberti, F. Lisacek, J.-C. Sanchez, and M. Müller. pROC: An open-source package for $\mathrm{R}$ and $\mathrm{S}+$ to analyze and compare ROC curves. BMC Bioinformatics, 12:77, 2011. [p44]

T. Sing, O. Sander, N. Beerenwinkel, and T. Lengauer. ROCR: Visualizing classifier performance in R. Bioinformatics, 21(20):3940-3941, 2005. [p44]

W. Velden M. \& Clark. Reduction of rating scale data by means of signal detection theory. Psychophysics, 25(6):517-518, 1979. doi:10.3758/BF03213831. [p44]

WHO. Mental disorders fact sheet. "www.who.int/mediacentre/factsheets/fs396/en/", April, 2016. [p53, 54]

Waldemar W. Koczkodaj

Computer Science

Ramsey Lk Rd. 
Laurentian University

Sudbury, Ontario P3E 2C6

Canada

wkoczkodaj@cs. laurentian.ca

http://cs. laurentian.ca/wkoczkodaj/info

Feng $L i$

Computer Science

Ramsey Lk Rd.

Laurentian University

Sudbury, Ontario P3E 2C6

Canada

spacejohn@live.cn

Alicja Wolny-Dominiak

Department of Statistical and Mathematical Methods in Economics

University of Economics in Katowice

ul. 1 Maja 50

40-287 Katowice

Poland

alicja.wolny-dominiak@uekat.pl

https://woali.github.io/rphdstatistics 